\title{
Prevenção da hipertensão e sua relação com o estilo de vida de trabalhadores
}

\author{
Prevention of hypertension and its relation to the lifestyle of workers \\ Prevención de la hipertensión y de su relación a la forma de vida de trabajadores
}

\author{
Maria Euridéa de Castro ${ }^{1}$, Maysa Oliveira Rolim² ${ }^{\text {,Tibelle Freitas Mauricio }}{ }^{3}$
}

\begin{abstract}
RESUMO: A hipertensão arterial é uma doença de fácil diagnóstico e com uma diversidade terapêutica eficaz para seu controle. Entretanto, grande parte dos hipertensos continuam ignorando-a, não controlando seus níveis tensionais, mesmo após diagnosticados. Dessa maneira, objetivou-se averiguar o conhecimento dos trabalhadores de uma Universidade Pública acerca do controle e da manutenção da pressão arterial dentro dos parâmetros normais e analisar a prática das medidas preventivas com vista à adoção de um estilo de vida compatível com o nível funcional ótimo. Foi realizado estudo descritivo com 32 trabalhadores de uma Universidade Pública Estadual em Fortaleza-Ceará. Utilizou-se um roteiro de entrevista semi-estruturado, sendo as falas categorizadas em unidades temáticas. Depreendeuse que os trabalhadores conhecem as mudanças de hábito a serem adotadas para prevenção da hipertensão. Relativo à obesidade, 26 (81\%) a consideram como fator de risco para a hipertensão. Quanto ao álcool e à raiva, $25(78 \%)$ e $17(53 \%)$, respectivamente, afirmaram serem indicativos para elevação da pressão arterial, bem como o fumo, citado por $23(72 \%)$ respondentes. Entretanto, a alimentação desses trabalhadores urge maior atenção, visto que 19 (60\%) não seguem uma dieta balanceada, tornando-se obesos ou com sobrepeso. Assim, notou-se que alterar hábitos é difícil, sobretudo os alimentares. Porém, a mudança de estilo de vida relaciona-se a ações educativas e à necessidade imperiosa de cada indivíduo frente aos seus problemas de saúde e sua resolução de querer atingir seu nível funcional ótimo.
\end{abstract}

Descritores: Hipertensão; Estilo de vida; Enfermagem

ABSTRACT: Arterial hypertension is an easily diagnosed illness and with efficient therapeutic diversity for its control. However, a large number of those affected continue to ignore it, not controlling their pressure levels even after diagnosis. In this way, the objective was to verify the awareness of workers at a public university concerning the control and maintenance of arterial pressure within normal parameters, and analyze the practice of preventative measures with a view to adopting a lifestyle compatible with an optimal functional level. A descriptive study was conducted with 32 workers of the Public State University in Fortaleza, Ceará, Brazil. The data and comments were categorized in thematic units. The conclusion was that the workers knew which changes of habits need to be adopted to prevent hypertension. With respect to obesity, $26(81 \%)$ considered it a risk factor for hypertension. Concerning alcohol and anger, $25(78 \%)$ and $17(53 \%)$ respectively agreed that they were indicative for a rise in arterial pressure. Similarly, smoking was mentioned by $23(72 \%)$ interviewees. Nevertheless, the eating habits of these workers demands more attention, as 19 (60\%) were not following a balanced diet, becoming obese or overweight. Thus it was observed that changing habits is difficult, especially eating habits. However, a change in lifestyle is related to awareness activities and the imperious needs of each individual with respect to his health problems and his determination to want to attain his optimal functional level.

Keywords: Hypertension; Life style; Nursing

RESUMEN: La hipertensión arterial es una enfermedad de fácil diagnóstico y con una diversidad terapéutica eficaz para su control. Sin embargo, gran parte de los hipertensos siguen ignorándola, no controlando sus niveles tensionales, incluso después de diagnosticados. De ese modo, se objetivó averiguar el conocimiento de los trabajadores de una Universidad Pública sobre el control y sobre el mantenimiento de la tensión arterial dentro de los parámetros normales y analizar la práctica de las medidas preventivas con miras a la adopción de un estilo de vida compatible con el nivel funcional óptimo. Se realizó un estudio descriptivo con 32 trabajadores de una Universidad Pública Estatal en Fortaleza, Ceará. Se procesó los datos y se categorizaron las hablas en unidades temáticas. Se percibió que los trabajadores conocen los cambios de hábito a ser adoptados para la prevención de la hipertensión. Con relación a la obesidad, 26 (el $81 \%$ ) la consideran como factor de riesgo para la hipertensión. En cuanto al alcohol y a la rabia, 25 (el $78 \%$ ) y 17 (el $53 \%$ ), respectivamente, afirmaron ser indicativos para la elevación de la tensión arterial, bien como el tabaco, citado por 23 (el $72 \%$ ) de los entrevistados. Sin embargo, la alimentación de esos trabajadores urge mayor atención, una vez que 19 (el $60 \%$ ) no siguen una dieta equilibrada, volviéndose obesos o con sobrepeso. Así, se percibió que alterar hábitos es difícil, sobre todo los alimentarios. No obstante, el cambio de estilo de vida está relacionado a acciones educativas y a la necesidad imperiosa de cada individuo frente a sus problemas de salud y su resolución de querer alcanzar su nivel funcional óptimo.

Descriptores: Hipertensión; Estilo de Vida; Enfermería

\footnotetext{
1 Docente Livre, Professora do Curso de Enfermagem da UECE. E-mail: eurideacastro@terra.com.br

2 Acadêmica do $8^{\circ}$ semestre de Enfermagem da UECE.

3 Acadêmica do ${ }^{\circ}$ semestre de Enfermagem da UECE.
} 


\section{INTRODUÇÃO}

O estado de saúde de um indivíduo pode ser influenciado pelo meio em que vive, por suas relações sociais, bem como por suas condições sócio-econômico-culturais, sendo precisamente indicado por sinais fisiológicos, entre eles a pressão arterial, cuja aferição deve ser feita por profissionais de saúde treinados e em toda avaliação clínica ${ }^{(1)}$.

A hipertensão arterial sistêmica (HAS) é uma doença crônico-degenerativa, cujo controle tem se tornado um desafio para os profissionais, visto que seu tratamento exige a participação ativa do hipertenso, no sentido de modificar alguns hábitos de vida prejudiciais à saúde e assimilar outros que beneficiem sua condição de saúde ${ }^{(2)}$.

A hipertensão é uma doença de alta prevalência no Brasil, atingindo os adultos jovens e os idosos em aproximadamente $20 \%$ e $50 \%$, respectivamente ${ }^{(3)}$. Em torno de $85 \%$ das pessoas que foram acometidas por acidente vascular encefálico e $40 \%$ das vítimas de infarto do miocárdio apresentam, como doença associada, a HAS. Dados do Instituto Nacional de Seguro Social (INSS) demonstram que $40 \%$ das aposentadorias precoces decorrem desse tipo de enfermidade ${ }^{(4)}$.

No entanto, mesmo com o fácil diagnóstico e a existência de uma grande diversidade terapêutica eficaz para o controle da hipertensão, grande parte dos milhões de brasileiros, que se estima possuírem a doença, continua ignorando-a e deixando de controlar seus níveis pressóricos $^{(5)}$.

Quanto à decisão terapêutica, deve-se considerar que o nível e a variabilidade da pressão arterial são fortemente influenciados por fatores genéticos associados a fatores ambientais ${ }^{(6)}$.

Dessa maneira, grande ênfase tem-se dado às medidas não farma-cológicas, de mudança no estilo de vida, para prevenção e controle dos níveis pressóricos elevados, que devem ser adotadas por todos os hipertensos, inclusive os farmaco-dependentes ${ }^{(7)}$, e por pessoas com forte antecedência familiar de hipertensão $0^{(8)}$.

Entre as mudanças que devem ocorrer na vida de um hipertenso, estão a redução do peso corporal, a dieta hipossódica e balanceada, o aumento da ingesta de frutas e verduras, a redução de bebidas alcoólicas, a realização de exercícios físicos, a cessação/atenuação do tabagismo e a substituição da gordura saturada por poliinsaturados e monoinsaturados. Sendo esses hábitos implementados, pode ser dispensada a terapia farmacológica ou a dose ou quantidade de drogas pode ser reduzida ${ }^{(8)}$.

Em relação aos fatores nutricionais, é fundamental a redução da ingesta calórica. Contudo, ao se intervir na consciência do paciente, transmitindo informações e corrigindo conceitos sobre a doença, consegue-se intervir positivamente contra a evolução dessa enfermidade ${ }^{(9)}$.
Relativo à atividade física, é conhecida por ter efeito benéfico sobre os fatores de risco tradicionais como elevação da pressão sanguínea, hiperinsulinemia e hiperlipidemia ${ }^{(10)}$.

A cada ano, mais de 2 milhões de mortes são atribuídas à inatividade física em todo o mundo. Essas mortes são parte do incremento de enfermidades, incapacidades e mortes causadas pelas doenças crônicas, entre elas as cardiovasculares $^{(11)}$.

O sal tem sido considerado, há muito tempo, um importante fator no desenvolvimento e na intensidade da hipertensão arterial, estando também relacionado ao aumento do risco para o desenvolvimento da hipertrofia ventricular esquerda, proteinúria e direta queda noturna da pressão. A sensiblidade ao sal está associada à mortalidade tanto em normotensos quanto em pessoas com hipertensão que possuem idade superior a $25 \operatorname{anos}^{(12)}$.

A condição sócio-econômica também pode ter influência multifa-torial na hipertensão. No mundo do trabalho, a hipertensão arterial sistêmica é mais encontrada entre os trabalhadores não-especializados, que ganham menores salários, dos setores secundários e terciários da economia. As pessoas menos favorecidas não possuem acesso a informações, não tendo, portanto, conhecimento sobre a hipertensão ${ }^{(13)}$.

Quanto ao tabagismo, é responsável por cerca de $45 \%$ das mortes nos homens com menos de 65 anos de idade e por mais de $20 \%$ de todos os óbitos por doença coronariana nos homens com idade superior a essa faixa etária. Além disso, considera que o cigarro, através da nicotina, aumenta a pressão arterial e leva a uma maior deposição de colesterol nos vasos sangüíneos ${ }^{(14)}$.

Dentro desse contexto, é de suma importância que o profissional de saúde, ao abordar um hipertenso, atente às percepções do paciente para que este venha a conhecer a sua doença, a desenvolver a auto-responsabilidade, a assumir seu papel ativo, a modificar seus comportamentos em relação à saúde e a manter sentimentos positivos ${ }^{(15)}$.

Nesse sentido, ressalta-se a importância da enfermagem no controle da doença crônica, principalmente com relação à educação, ao encorajamento e ao monitoramento do indivíduo, a fim de promover melhorias no seu estado geral ${ }^{(16)}$.

A respeito da promoção à saúde, são fundamentais as ações direcionadas à educação e à prática de prevenção dos fatores de risco, já que se pretende vislumbrar uma boa qualidade de vida à população $0^{(17)}$.

Dessa forma, promover a saúde implica auxiliar as pessoas a terem hábitos saudáveis. No entanto, a mudança de estilo de vida é melhor obtida quando o indivíduo com hipertensão se acha constantemente estimulado ao longo do acompanhamento ${ }^{(4)}$.

Assim, sabendo que a hipertensão é uma enfermidade multisistêmica e multifatorial, sendo necessário que 
o indivíduo adapte-se à cronicidade da doença, considerou-se oportuno estudar este tema, com o objetivo de averiguar o conhecimento dos trabalhadores de uma Universidade Pública acerca do controle e da manutenção da pressão arterial dentro dos parâmetros normais, bem como analisar a prática das medidas preventivas imple-mentadas com vista à adoção de um estilo de vida compatível com o nível funcional ótimo.

\section{MÉTODO}

O estudo é de natureza descritivo-exploratória, realizado com um grupo de trabalhadores lotados no Restaurante Universitário e nos Serviços de Apoio de uma Universidade Pública Estadual da Cidade de Fortaleza-Ceará, no período de março de 2002 a fevereiro de 2003.

O universo do estudo foi constituído por 32 trabalhadores que aceitaram participar do estudo e assinaram o termo de consentimento livre e esclarecido. O número de indivíduos a constituírem a amostra da pesquisa não foi estabelecido previamente e a amostra foi aleatória simples, constituída a partir do interesse dos autores em atuarem nos setores de maior predomínio de indivíduos portadores de hipertensão arterial.

A escolha dos setores deu-se a partir de um sub-projeto acerca da hipertensão anteriormente realizado entre docentes e funcionários da universidade em evidência, o qual constatou uma freqüência maior de pessoas com hipertensão nos referidos setores.

O critério de seleção da amostra foi a inclusão de todos os indivíduos lotados nos setores em estudo no momento da coleta de dados, independente de terem ou não problemas de saúde relacionados ao controle da pressão arterial. Desse modo, todos os indivíduos presentes foram incluídos no estudo, respeitando-se a liberdade e o livre arbítrio em participar da pesquisa.

Utilizou-se, como instrumento de coleta de dados, um roteiro de entrevista semi-estruturado, gravado em fita K-7, o qual constou de duas partes: a primeira sobre caracterização clínico-demográfica e sobre os fatores intervenientes para hipertensão, entre eles: idade, sexo, raça, escolaridade, peso, tabagismo, etilismo, antecedentes pessoais e familiares, e a segunda composta por perguntas abertas acerca dos fatores intervenientes para a hipertensão arterial.

Os dados gravados foram transcritos com fidedignidade, e após transcrição completa das entrevistas, seguiu-se a Análise de Conteúdo de $\operatorname{Bardin}^{(18)}$, sendo as falas categorizadas, por similitude, em unidades temáticas, a saber: a obesidade e sua relação com a hipertensão; o efeito do álcool sobre a pressão arterial; sal e hipertensão; a raiva e sua interferência na pressão; fumo $X$ hipertensão.
O projeto foi aprovado pelo Comitê de Ética da Universidade Estadual do Ceará (UECE), conforme a Resolução ${ }^{(19)} 196 / 96$, e, aos respondentes, foram assegurados o sigilo das informações, o anonimato e o livre-arbítrio na inclusão do estudo. Foram informados, ainda, sobre o Termo de Consentimento Livre-Esclarecido que, ao final, foi assinado pelos participantes.

\section{RESULTADOS E DISCUSSÃO}

Quanto à caracterização sócio-demográfica e clínica, depreendeu-se que os entrevistados possuem a seguinte constituição: 20 (63\%) são do sexo masculino, 25 (78\%) pertencem à faixa etária de 40 anos ou mais, 14 (44\%) possuem o ensino fundamental incompleto, $23(72 \%)$ têm renda familiar entre 3 e 7 salários mínimos, $13(41 \%)$ apresentam antecedentes familiares de HAS e 11 (34\%) de diabetes, 13 (41\%) estão com sobrepeso, 5 (16\%) com obesidade leve e $1(3 \%)$ com obesidade moderada, $7(22 \%)$ fazem uso do cigarro, 11 (34\%) são etilistas e 11 (34\%) respondentes são portadores de hipertensão.

As falas dos respondentes resultaram nas unidades temáticas em destaque:

\section{Obesidade e sua relação com a hipertensão}

Relativo a essa temática, pôde-se detectar que 26 $(81 \%)$ respondentes consideram a obesidade um fator de risco para a hipertensão, visto que o excesso de peso pode acarretar a elevação da taxa de colesterol e exige maior esforço do indivíduo para realização de tarefas cotidianas.

Observou-se ainda que associam a obesidade ao aumento da pressão arterial devido a casos ocorridos com amigos, bem como a orientações médicas, conforme depoimentos:

Eu acho que aumenta, né? A pessoa gorda tem tendência a gordurar o sangue, né? Eu tenho uma sobrinha que ela tem 25 anos e pesa $140 \mathrm{Kg}$, horrível de gorda, ela já foi no médico e o médico disse que ela tem problema de pressão. (E.18)

Eu acredito que sim, porque a primeira orientação de quem tem problema de pressão alta é exatamente o cuidado na educação alimentar, deve ter uma relação direta. (E.20)

Acho que interfere na pressão, porque uma pessoa com um peso muito elevado ela se esforça mais para se movimentar... aí aumenta a pressão. (E.29) 
Apesar da obesidade ser mais freqüente em mulheres, é nos homens que ocorrem as maiores complicações relacionadas à hipertensão e ao excesso de peso. A distribuição da gordura no sexo feminino é, predominantemente, na região glútea e áreas anexas, não causando grandes riscos à saúde, enquanto a gordura depositada no abdome, comum nos homens, propicia o surgimento da hipertensão $\operatorname{arterial}^{(20)}$.

Uma redução de 5\% a $10 \%$ no peso corporal é capaz de promover reduções significativas na pressão arterial, à medida que o peso leva à natriurese com menor atividade do sistema nervoso simpático e à redução da insulinemia ${ }^{(21)}$.

\section{O efeito do álcool sobre a pressão arterial}

Relativamente ao efeito do álcool sobre a pressão arterial, $25(78 \%)$ respondentes afirmaram ser essa substância um risco para o aumento pressórico, na medida que eleva a temperatura do corpo e aumenta o metabolismo, prejudicando não somente o sistema cardíaco, mas também os outros sistemas fisiológicos.

Entretanto, houve também aqueles que referiram a maleficência do consumo de álcool associando-a a experiências próprias. Observe os depoimentos.

A bebida alcoólica é péssima, aumenta tudo...tem efeito nocivo, ele influi no seu sistema nervoso e no seu organismo, ele influi na sua eliminação de urina, no seu sistema digestivo, urinário, é... total, é ruim em todos os sistemas.(E.24)

Como interfere na pressão! Porque assim, ele eleva a pressão, a temperatura do álcool muda no corpo e agita o corpo, a pressão sobe... (E.29)

...todo desmantelo do meu organismo eu só culpo a bebida (...) todas as consequiências que eu tenho na minha saúde pra mim o único culpado é a bebida...(E.16)

O álcool tem também seu efeito sobre a pressão arterial, sendo mais intenso no branco e idoso que no negro e no jovem, causando redução na resistência vascular periférica e aumentando o débito cardíaco. Por isso, recomenda-se como limites de consumo aceitáveis, para os homens, aproximadamente $720 \mathrm{ml}$ de cerveja, $60 \mathrm{ml}$ de bebida destilada e $240 \mathrm{ml}$ de vinho. Porém, àqueles indivíduos que não conseguem se enquadrar nesse limiar, se sugere o abandono do consumo de bebidas alcoólicas ${ }^{(1)}$.

\section{Sal e hipertensão}

Nesta unidade temática, denotou-se que 21 (66\%) respondentes associam o sal à elevação da pressão arte- rial. No entanto, alguns acreditam que o efeito maléfico desse mineral seja a longo prazo, podendo comprometer a saúde apenas com o avançar da idade. Entretanto, observou-se uma certa preocupação quanto ao uso de uma dieta hipossódica, conforme os depoimentos:

A alimentação com certeza eu acho que interfere na pressão, por exemplo, se a pessoa come salgado vai com certeza alterar a pressão dela. No meu caso, como eu não tô hipertensa, eu tenho a pressão baixa, eu posso abusar do sal, mas uma pessoa hipertensa não pode...eu acho que se eu não moderar o sal, talvez, no decorrer da minha vida, quando eu fizer uns 40 anos, talvez eu possa ser hipertensa. (E.23)

Eu evito as coisas que eu sei que são carregadas de sal e procuro evitar porque eu tenho preocupação... a partir de agora eu tô na terceira idade... se não tiver cuidado aos 50 anos, não vou chegar aos 80. (E.20)

Ainda relacionado ao efeito desse mineral sobre a pressão arterial, notou-se que alguns respondentes não possuem o apoio familiar na mudança desse hábito de vida, visto que recebem críticas a respeito da mudança no tipo de alimentação. Observem o depoimento:

...aumenta a pressão, minha comida lá em casa é tudo insosso, assim, não é muito...meus filhos falam: passa o sal pra cá mãe, que a mãe hoje... aí eu: só que vocês são de uma média eu sou de outra, toma o sal todinho pra vocês... eu tenho que manerar, meu marido é diabete e tem pressão alta... (E.12)

Quanto à ingesta de sal, considera-se que abolir diariamente da dieta $10 \mathrm{~g}$ a $5 \mathrm{~g}$ desse mineral reduz a pressão arterial em 5/3 mmHg, sendo esse efeito maior em pessoas idosas e em indivíduos em estágio inicial de elevação dos níveis tensionais ${ }^{(22)}$.

Quanto a isso, os indivíduos podem ser divididos em sensíveis e não sensíveis ao sal, sendo recomendado, apesar da resposta heterogênea a restrição de sódio, uma ingestão de sal menor que $6 \mathrm{~g} /$ dia ou cerca de 2,3g de sódio ${ }^{(23)}$.

Estando a sensibilidade ao sal associada a um posterior desenvolvimento de hipertensão, é possível que a intervenção em indivíduos susceptíveis, através da redução da ingesta de sal, possa prevenir ou retardar conseqüentes aumentos da pressão devido à idade, ao de-senvolvimento da hipertensão e ao aumento do risco para doenças cardiovasculares ${ }^{(12)}$.

\section{A raiva e sua interferência na pressão}

Relativamente à raiva como fator de risco para HAS, $17(53 \%)$ respondentes afirmaram conhecer tal interfe- 
rência, associando este fator ao relacionamento interpessoal e ao estresse no ambiente de trabalho, sendo por isso que procuram ter uma boa interação com as pessoas. Observem os depoimentos.

...a raiva aumenta também a pressão, porque quando você tem raiva mexe com tudo, né?[...] quando você tá tranqüilo a pressão tá normal, tá tudo bem, eu tiro por mim né? quando tá tudo bem no trabalho, lá em casa aí tá tudo bem... (E.4)

...é isso que eu quero fazer (evitar a raiva), qualquer coisinha eu me agito...eu tô até um pouco vermelho, não tô? Quando eu tô normal eu não fico assim, não! (E.10)

... eu não discuto com ninguém e quem discutir comigo eu vou me embora, porque se eu discutir vou alterar minha pressão, vou ter raiva e a raiva deixa a pressão do cara lá em cima, sem dúvida... (E.30; E.31)

O estresse mental é outro fator de risco adicional para a doença hipertensiva, visto que uma exagerada resposta cardiovascular às situações estressantes tem mostrado estar associado à isquemia do miocárdio, e é um prognóstico para um futuro desenvolvimento da hipertensão e doenças coronárias ${ }^{(10)}$.

Um aspecto essencial da manutenção da saúde é tentar manter a harmonia nos relacionamentos, sejam familiares, de trabalho ou de amizade. Existem indivíduos que constroem relacionamentos de brigas constantes, e outros que ficam submersos em tristeza, propiciando a formação de tensões crônicas, que pioram a qualidade de vida e são a base de inúmeras doenças ${ }^{(24)}$.

\section{Fumo X hipertensão}

Referente ao tabagismo, 23 (72\%) relataram o prejuízo causado pelo fumo à saúde, mais particularmente aos pulmões e à pressão arterial. Entretanto, associaram tal influência a experiências próprias ou de familiares e amigos, bem como à ação da nicotina. Relataram ainda acerca do tabagismo passivo, conforme se nota nos depoimentos destacados.

Rapaz, o fumo é o mais cruel pra saúde da gente, porque faz mal. Toda pessoa que fuma tem mau hálito[...] tem aquele sarro, aquele catarro no peito que nunca larga, você dificilmente vê um fumador que não tosse... o fumo faz mal, né? Ele faz mal pra pressão e para outros tipos de coisa... eu fumei desde os meus 15 anos, aí eu vi que não dava certo... (E.4)
O fumo interfere muito, né? Com certeza faz mal à saúde, né?Eu fumei muito quando pequeno, mas era porque meus pais [...] através deles fumarem a gente fuma também, né? sem querer, mas eu nunca utilizei nem fumo nem cigarro. (E.11)

...o fumo altera demais a pressão, porque tem a nicotina [...] várias pessoas que fumam têm problema cardíaco, eu conheço pessoas que quando pararam de fumar ficaram melhor. (E.15;22)

O hábito de fumar é um dos principais fatores de risco para as doenças cardiovasculares, devendo ser, então, abolido. Porém, a dependência à nicotina, hoje considerada uma droga que provoca vício igual ao da cocaína e heroína, dificulta o seu abandono ${ }^{(4)}$.

$\mathrm{O}$ fumo, entre outras maneiras de contribuir para o desenvolvimento das doenças coronarianas, deflagra a liberação de catecolaminas, que elevam a freqüência cardíaca e a pressão arterial, através do ácido nicotínico presente no tabaco ${ }^{(25)}$.

Os fumantes apresentam um aumento de 10 vezes o risco de morte cardíaca súbita, sendo que aquelas pessoas que pararam de fumar têm seu risco de cardiopatia diminuído em $30 \%$ a $50 \%$ dentro do primeiro ano, tendo esse risco em contínuo declínio enquanto elas se abstiverem de fumar ${ }^{(25)}$.

\section{CONSIDERAÇÕES FINAIS}

Pôde-se depreender que os trabalhadores investigados têm conhecimento da mudança de hábito a ser adotada para prevenção da hipertensão, como a adoção de uma dieta balanceada e hipossódica, visando manter o equilíbrio do peso corporal e dos níveis de sódio. Entretanto, o estilo de vida desses trabalhadores urge uma maior preocupação a respeito da alimentação, visto que $19(59 \%)$ não obedecem uma dieta balanceada, tornando-se obesos ou com sobrepeso.

A respeito do etilismo e tabagismo, 21 (67\%) e 25 (78\%) trabalhadores, respectivamente, procuram, muitas vezes, evitá-los. Dessa forma, pôde-se observar uma maior conscientização destes quanto aos males causados por tais vícios.

O controle da ingesta de sal também é uma precaução de 21 (66\%) trabalhadores pesquisados, embora se tenha observado a importância do apoio familiar para essa mudança de hábito de vida. À medida que se tem a ajuda dos familiares e amigos, a aquisição de um novo estilo de vida é estimulada, favorecendo a redução dos riscos para hipertensão.

O bom relacionamento interpes-soal no local de trabalho é outra medida utilizada por 17 (53\%) trabalhadores a fim de prevenir emoções negativas, como a raiva, 
as quais produzem um efeito nocivo sobre o organismo, mais particularmente sobre os vasos sanguíneos, quando ocorridos com freqüência.

Dessa maneira, alterar hábitos é tarefa difícil, sobretudo os alimentares. Porém, a mudança de estilo de vida está relacionada a ações educativas e a necessidade imperiosa de cada indivíduo frente aos seus problemas de saúde e a sua resolução de querer atingir seu nível funcional ótimo.

É neste contexto que o enfermeiro, como membro de uma equipe multiprofissional, tem um papel especial. É incontestável a necessidade de o enfermeiro ter que priorizar a compreensão do quê o indivíduo pensa, integrando-o ao seu contexto social e cultural, proporcionando que este reflita e desencadeie uma atitude crítica acerca da sua real situação de saúde e de vida.

\section{REFERÊNCIAS}

1. Sociedade Brasileira de Hipertensão. Sociedade Brasileira de Cardiologia. Sociedade Brasileira de Nefrologia. $4^{\text {a }}$ Diretrizes Brasileiras de Hipertensão Arterial. Campos do Jordão; 2002.

2. Cade NV. A teoria do déficit de autocuidado de Orem aplicada em hipertensas. Rev Lat Am Enferm 2001; 9(3):43-50.

3. Oliveira TC, Araújo TL, Melo EM, Almeida DT. Avaliação do processo adaptativo de um idoso portador de hipertensão arterial. Rev Lat Am Enferm 2002; 10(4):530-36.

4. Conselho Nacional de Saúde. Resolução 196 de 10 de outubro de 1996: Diretrizes e normas regulamentadoras da pesquisa envolvendo seres humanos. Brasília: Ministério da Saúde; 1996.

5. Bloch KV, Klein CH, Souza e Silva NA, Nogueira AR, Campos LHS. Hipertensão arterial e obesidade na Ilha do Governador - Rio de Janeiro. Arq Bras Cardiol 1994; 62(1):17-22.

6. Rocha E, Porto M, Morelli MYG, Maestá N, Waib PH, Burini RC. Efeito de estresse ambiental sobre a pressão arterial de trabalhadores. Rev Saúde Pública. 2002; 36(5):568-75.

7. Silva Junior PCS, Martins RCA, Dantas EHM. Os efeitos da atividade física na prevenção da hipertensão. Rev Bras Med Esporte1999; 5(2):66-72.

8. Harbron E. Medical and complementary. Nurs Times 2002; 98:32-4.

9. Oigman W. Recomendações não farmacológicas coadjuvantes no tratamento da hipertensão arterial. Arq Bras Cardiol 1991; 57(4):341-6.

10. Georgiades A, Sherwood A, Gullette EC, Babyak MA, Hinderliter A, Waugh R et al. Effects of exercise and weight loss on mental stress- induced cardiovascular responses in individuals with high blood pressure. Hypertension. 2000; 36(2):171-6.
11. Matsudo VKR. Promoção da saúde mediante o aumento do nível de atividade física: proposta do programa Agita São Paulo. Rev Âmb Med Esportiva 2001:5-15.

12. Weinberger MH, Fineberg NS, Fineberg SE, Weinberger M. Salt sensitivity, pulse pressure, and death en normal and hypertensive humans. Hipertension. 2001;37(2 Part 2): 429-32.

13. Cordeiro R. Hipertensão e Ocupação: algumas considerações a respeito da epidemiologia da hipertensão arterial sistêmica entre os trabalhadores. Saúde Deb 1991; (32):53-7.

14. Instituto Nacional do Câncer (INCA). Manual falando do tabagismo. [online]. Rio de Janeiro; 2002. Disponível em: <http://www.falandoseriosobredrogas.org.br/tabcurso.htm>. (2 jan. 2003).;

15. Reis MG, Glashan RQ. Adultos hipertensos hospitalizados: percepção de gravidade da doença e de qualidade de vida. Rev Lat Am Enferm 2001; 9(3):51-7.

16. Coady R. Cronic heart failure. Nurs Times 2002; 98:41-4.

17. Nascimento LC, Mendes IJM. Perfil de saúde dos trabalhadores de um Centro de Saúde-Escola. Rev Lat Am Enferm 2002; 10(4):502-8.

18. Bardin L. Análise de conteúdo. Rio de Janeiro: Edições 70; 1977.

19. Ministério da Saúde. Departamento de Atenção Básica. Área Técnica de Diabetes e Hipertensão. Hipertensão Arterial Sistêmica (HAS) e Diabetes Mellitus (DM): protocolo. Brasília: Ministério da Saúde; 2001.

20. Moreira TMM, Maciel ICF, Araújo TL. Trabalhando a autoajuda em Grupo no Controle da Hipertensão. Cardiol Nurs 1999; 2:20-4.

21. Plavnik FL, Kohlmann Junior O. Hipertensão arterial: como diagnosticar e tratar. Rev Bras Med 2002; 59(1/2):49-60.

22. Hurst R. Managing hipertension: measurement and prevent. Nurs Times 2002; 98:38-40.

23. Mano R. Tratamento não-farmacológico da hipertensão arterial. Manual da hipertensão arterial. [online] 2002. Disponível em: <http://www.manuaisdecardiologia.med.br/has/Pag1.htm>. (2 jan. 2003).

24. Maldonado MT, Goldin A. Maioria de 40: guia de viagem para a vida. São Paulo: Saraiva; 1997. Estilo de vida: mudando a maneira de olhar; p. 30-41.

25. Smeltzer SC, Bare BG. Brunner \& Studart: tratado de enfermagem médico-cirúrgica. $9^{\mathrm{a}}$ ed. Rio de Janeiro: Guanabara Koogan; 2002. Tratamento de pacientes com distúrbios vasculares coronarianos; p. 572-5. 